\title{
Editorial: Antimicrobial Nanostructured Polymeric Materials and Nanocomposites
}

\author{
Magdalena M. Stevanovic ${ }^{1 *}$, Marija Vukomanovic ${ }^{2}$, Marina Milenkovic $^{3}$ and \\ Aldo R. Boccaccini ${ }^{4}$ \\ ${ }^{1}$ Institute of Technical Sciences of the Serbian Academy of Sciences and Arts, Belgrade, Serbia, ${ }^{2}$ Advanced, Materials \\ Department, Jožef Stefan Institute, Ljubljana, Slovenia, ${ }^{3}$ Department of Microbiology and Immunology, Faculty of Pharmacy, \\ University of Belgrade, Belgrade, Serbia, ${ }^{4}$ Department of Materials Science and Engineering, Institute of Biomaterials, University of \\ Erlangen-Nuremberg, Erlangen, Germany
}

Keywords: nanostructured materials with antimicrobial activity, mechanisms of action of antimicrobial nanomaterials, role of antimicrobial nanomaterials, toxicity and adverse effects, future perspectives

Editorial on the Research Topic

Antimicrobial Nanostructured Polymeric Materials and Nanocomposites

\section{INTRODUCTION}

Infectious diseases are a significant burden on the public health and the economic stability of societies all over the world. Drug resistance presents an ever-increasing global public health threat that involves all major microbial pathogens and antimicrobial drugs. The considerable concern regarding bacterial resistance to antimicrobial agents, bacterial adhesion, and proliferation has generated intense research in this field. Although urgently needed, an effective and long-lasting solution to this problem, i.e. microbial and especially bacterial colonization, has not yet been found. In the last decades, nanomaterials have attracted much attention because of their unique physical, chemical, and mechanical properties, due to their high surface area and size at the nanoscale. Owing to these extraordinary characteristics, nanomaterials are appropriate candidates for various applications in different fields such as medicine, pharmacy, food industry, etc. New strategies based on the use of nanomaterials such as nanoparticles, nanocapsules, nanogels, nanofibers, nanocomposites, or nanocoatings for controlling microbial virulence factors are very promising. In addition special focus recently is paid on nanostructured polymeric materials and nanocomposites with antimicrobial properties. The increasing attention within the scientific community regarding this topic can be easily assessed by searching the suitable keywords in some recognized literature databases. According to Scopus, in the last 10 years, more than 1,300 peer-reviewed documents have been published jointly containing keywords such as "nanocomposites" and "antimicrobial". Interestingly, in the more specified search which refers to the utilization of nanostructured polymeric materials, it could be observed a similar profile i.e. growing tendency.

\section{Description of the Special Issue}

This Special Issue provides selected contributions on advances in the synthesis, characterization, and applications of nanocomposites, nanofibers, nanoparticles, nanofiber membranes, but also innovative macroporous and mesoporous geopolymers inspired by adsorption properties of naturally-occurring aluminosilicates as well as strategies to manage microbial composition by the formation of biofilms with beneficial microbes. 
Metal and metalloid nanoparticles are very popular antimicrobials since they manifest non-specific bacterial toxicity mechanisms which makes the development of resistance by bacteria to be difficult. Abbas et al. have described obtaining iron oxide/zinc oxide nanocomposite by an ecofriendly green method for the synthesis of iron oxide nanoparticles and iron oxide/ $\mathrm{ZnO}$ nanocomposite using the phytochemicals. Iron oxide nanoparticles were amorphous, spherical, and irregular in shape while the iron oxide/ZnO nanocomposite showed rod-shaped $\mathrm{ZnO}$ surrounding amorphous iron oxide nanoparticles. The high antimicrobial activity was demonstrated in the case of iron oxide nanoparticles against MRSA and E. coli and this was not the case with iron oxide nanocomposite. The possible explanation of the authors is that iron oxide nanocomposite does not show antimicrobial activity due to the particle instability and their negative surface charge that was not able to affect the negatively charged cell wall of bacterial cells implying that cationic surface would be a much effective solution. The last hypothesis was very effectively confirmed by Filipović et al. They synthesized different formulations of selenium nanoparticles by chemical reduction method using different stabilizers and reducing agents. The authors evaluated the effects of selenium nanoparticles' surface chemistry and structure on antimicrobial activity against eight standard bacterial strains and one yeast strain (S. aureus, E. faecalis, $B$. subtilis, K. rhizophila, E. coli, S. Abony, K. pneumoniae, and P. aeruginosa, $C$. albicans). All selenium nanoparticles were amorphous, spherical, and with sizes of about $75-200 \mathrm{~nm}$ but exhibited different antimicrobial activity, as discussed in the paper. The particle form, size, and surface chemistry are very important parameters that affect the interaction of nanomaterials with biological entities.

Polymers could be designed as very effective carriers of antimicrobial agents. In the study of Liu et al. newly synthesized composite nanofibers showed significantly better antibacterial properties against strains of $S$. aureus and $P$. aeruginosa compared with blank nanofibers. Liu et al. reported the synthesis of nanofibers with different ratios of poly (vinyl alcohol) and chitosan incorporated with moxifloxacin hydrochloride. The synthesis was performed by the blending electrospinning method. Moxifloxacin hydrochloride was loaded at high efficacy (95.38\%) on the nanofibers and formed hydrogen bonding with poly (vinyl alcohol) and chitosan. Yu et al. also used the electrospinning method but for the preparation of chitosan (CS)-polyethylene oxide (PEO) nanofiber membranes containing different concentrations of an antibacterial peptide NP10. The authors evaluated releasing of NP10 from the nanofiber membranes as well as antibacterial activity and biocompatibility of the samples.

In the study of Popovich et al. cost-effective, synthetic aluminosilicate geopolymers with controllable mechanical properties, porosity, and surface characteristics were produced. This work is inspired by the adsorption properties of naturally occurring aluminosilicates which are capable to remove harmful toxins from the environment. The authors showed that these laboratory-synthesized geopolymers adsorbed and physically removed several adsorbates, including large pore-forming toxins, MRSA proteins, and viable MRSA cells, from solutions with different efficacies. They concluded these materials could serve as cost-effective aluminosilicates for biomedical applications, physically removing harmful toxins and cells from infectious wound sites.

Current strategies to manage biofilms that can damage materials are focused on inhibition and removal of the biofilm. However, Deev et al. in their work propose instead of biofilm prevention, management of microbial composition by the formation of biofilms with beneficial microbes. They used an electrostatic modification of the surfaces of cells or the material by positively charged acetylated polyelectrolytes (PE) to enable efficient attachment of viable bacterial cells. The authors showed that artificial biofilms with extended stability can be constructed in this way.

\section{CONCLUSION}

This Special Issue on antimicrobial nanomaterials brings together experts from nanotechnology, materials science, physical chemistry, microbiology, among others, who have contributed cutting-edge results on this topic. The number of papers in the field constantly increases, indicating an urgent need for improving existing technologies and for the development of new promising solutions for the prevention, control, or elimination of infections exploiting nanomaterials.

\section{AUTHOR CONTRIBUTIONS}

MS: Conceptualization, Writing-Original draft preparation; MV, $\mathrm{MM}$, and $\mathrm{AB}$ : Writing-Reviewing and Editing.

\section{FUNDING}

This work was supported by the Ministry of Education, Science and Technological Development of the Republic of Serbia (Agreement on realization and financing of scientific research work of the Institute of Technical Sciences of SASA in 2021 (Record number: 451-03-9/2021-14/200175), and as a part of bilateral collaboration between the Republic of Serbia and Germany funded by the German Academic Exchange Service (DAAD project number 57514776).

Conflict of Interest: The authors declare that the research was conducted in the absence of any commercial or financial relationships that could be construed as a potential conflict of interest.

Publisher's Note: All claims expressed in this article are solely those of the authors and do not necessarily represent those of their affiliated organizations, or those of the publisher, the editors and the reviewers. Any product that may be evaluated in this article, or claim that may be made by its manufacturer, is not guaranteed or endorsed by the publisher.

Copyright (c) 2021 Stevanovic, Vukomanovic, Milenkovic and Boccaccini. This is an open-access article distributed under the terms of the Creative Commons Attribution License (CC BY). The use, distribution or reproduction in other forums is permitted, provided the original author(s) and the copyright owner(s) are credited and that the original publication in this journal is cited, in accordance with accepted academic practice. No use, distribution or reproduction is permitted which does not comply with these terms. 